\title{
Instrumental reversal learning and subsequent fixed ratio performance on simple and go/no-go schedules in neodecorticate rabbits
}

\author{
DAVID A. OAKLEY \\ Medical Research Council Unit on Neural Mechanisms of Behavior \\ 3 Malet Place, London WC1E 7JG, England
}

\begin{abstract}
Neodecorticated rabbits (94\%-97\% of neocortex removed) were able to acquire and repeatedly reverse an instrumental two-treadle differentiation based on food reward. Completion of 15 reversals of the two-treadle differentiation by the neodecorticates was followed by successful training on fixed ratio (FR) schedules as high as FR 60. The neodecorticates' rate of treadle pressing on FR was lower than normals', but efficiency was high and food-tray-related behaviors did not intrude on the treadle-press response. The improvements in FR performance in these neodecorticates in comparison with those in previous studies was interpreted as a result of enhanced manipulandum identification produced by the prior reversal training. The neodecorticates were impaired, however, in their ability to form reversal learning sets and in the establishment of go/no-go performance on a FR baseline. Possible interpretations of the data in terms of Pavlovian and instrumental learning and the differential representation of these two processes in the brain were considered.
\end{abstract}

The use of surgical ablation techniques to investigate the role of neocortex in adaptive behavior has a long history (Goltz, 1892) and has led to a variety of interpretations (Berlucchi \& Buchtel, 1975; Buchwald \& Brown, 1973). Pavlov (1927) proposed that all forms of conditioning and learning in mammals were dependent on neocortex. This belief was perpetuated by many of his students (Lebedinskaia \& Rosenthal, 1935), though other workers went on to demonstrate a variety of conditional responses in neodecorticate preparations both in the Soviet Union (Zeliony \& Kadykov, 1938) and in the United States (Culler \& Mettler, 1934; Girden, Mettler, Finch, \& Culler, 1936). Bromiley (1948) was able, by careful and prolonged testing of his decorticate dog, to show discrete leg flexion responses under what appear to have been shock-avoidance conditions. While the latter may have been taken as evidence of simple instrumental learning in a decorticate, the general conclusion drawn from these early studies plus later work involving cortical spreading depression was that Pavlovian conditioning was not dependent upon intact neocortex whereas instrumental learning was (Oakley \& Russell, 1972; Russell, 1966, 1971). This view was supported by the elegant experiments of DiCara, Braun, and Pappas (1970) which demonstrated that curarized, decorticated rats could learn autonomic responses when the training paradigm was Pavlovian but not when it was instrumental, despite identical sensory and motor demands in the

Present address: Department of Psychology, University College London, Gower Street, London, WC1E 6BT, England. two conditions. Recent work using the nictitating membrane response in neodecorticate rabbits has confirmed that areas of the mammalian brain outside neocortex are adequate substrates for Pavlovian conditioning, though slightly more trials were needed to initiate this process in the lesioned animals (Enser, 1976; Oakley \& Russell, 1975, 1976) and conditional response onset latencies remain slightly longer in the decorticates than in the controls (Enser, 1976; Oakley \& Russell, 1975, 1976). Differentiation and reversal learning using the Pavlovian paradigm, on the other hand, appear to be clearer in neodecorticates than in normals (Oakley \& Russell, 1974, 1975, 1976) unless subcortex is also damaged (Enser, 1976). The fact that a differential (light/tone) Pavlovian nictitating membrane response is not lost when normal training is followed by complete neodecortication (Oakley \& Russell, 1977) suggests that neocortex may not be involved in Pavlovian conditioning of this sort even in the intact animal-or at least that a parallel record of the entire transaction is available in subcortex. These data, then, strengthen the conviction that subcortex is a perfectly adequate, and may even be the normal, substrate of Pavlovian conditioning and are consistent with earlier failures to find a mass action effect on Pavlovian conditioning due to subtotal neocortical removal in similar situations (Oakley \& Russell, 1968, 1972).

The question of instrumental learning in the absence of neocortex remains to be considered. From a comparative point of view, it is clear that quite complex forms of instrumental learning can be demonstrated not only in those vertebrates (fish, 
amphibians, reptiles, and birds) which possess little or no true neocortex (Bitterman, 1965; Flood, Overmier \& Savage, 1976; Warren, 1974), but also in invertebrates which have quite differently organized central nervous systems (McConnell \& Jacobson, 1974). In mammals, it could be argued, however, that encephalization of function has occurred during phylogeny, with the result that subcortical structures have surrendered their ability to mediate instrumental learning to neocortex. This would be consistent with those earlier studies which have shown instrumental learning to be absent (Bloch \& Lagarrigue, 1968; DiCara, Braun, \& Pappas, 1970), or at best rudimentary and fragile (Bromiley, 1948), when neocortex is completely removed surgically. It is not, however, entirely consistent with other studies which have demonstrated instrumental learning in striatal cats (Bjursten, Norrsell, \& Norrsell, 1976) as well as in thalamic (Huston \& Borbély, 1974) and spinal rats (Chopin \& Buerger, 1975), nor is it consistent with recent observations of treadle pressing for food in totally neodecorticate rabbits (Oakley, 1971; Oakley \& Russell, 1978a, 1978b). One possible reason for successful instrumental learning in the latter studies may be the use of food reinforcement. All the earlier negative studies cited above which limited their lesions to neocortex used shock as the instrumental reinforcer (Bloch \& Lagarrigue, 1968; Bromiley, 1948; DiCara, Braun, \& Pappas, 1970). In line with this suggestion, instrumental learning in partially neodecorticated rats (Bloch \& Bello, 1974) and striatal cats (Bjursten, Norrsell \& Norrsell, 1976) has been observed more readily when food reinforcement was used instead of shock avoidance.

Though free-operant behavior has now been shown in neodecorticates, it is distinguished by its inefficiency in comparison to that of normal animals (Oakley, 1971; Oakley \& Russell, 1978a, 1978b). In particular, it has not been possible to demonstrate fixed ratio (FR) performance above FR 8 in the absence of neocortex and the lesioned animals tend to incorporate behaviors related to the food tray with the treadle-pressing sequence, making a rather global operant response. This contrasts with the clear separation which normal animals maintain between manipulandum and food-tray-related behaviors in the same situation. One suggestion has been that the decorticates fail to identify the manipulandum or to distinguish it adequately from the food tray. The most recent study (Oakley \& Russell, 1978b) indicated that if the lesioned animals were pretrained on a manipulandum situated at some distance from the food tray, their subsequent FR performance with the manipulandum returned to the vicinity of the food tray was more rapidly acquired and more efficiently executed. Pretraining procedures of this sort may serve to enhance the distinctiveness of the manip- ulandum for the decorticate and so improve its ability to treat the various components of its behavior separately in relation to reinforcement. The present study uses a two-treadle manipulandum discrimination and reversal procedure as a means of enhancing manipulandum identification by neodecorticates, and evaluates the effect of this pretraining on subsequent FR performance under simple and multiple (FR 10: extinction) schedules.

\section{EXPERIMENT 1}

In an attempt to ensure that the neodecorticated and normal animals identified the manipulandum, they were first of all trained to discriminate between two similar response treadles in a free-operant situation and to then repeatedly reverse that differentiation. This serves both as a pretraining procedure, to be evaluated in Experiment 2, and as an investigation of differentiation and the development of reversal learning sets in the absence of neocortex. Partially neodecorticated rats $(41.1 \%-68.2 \%$ of neocortex removed) have been shown to perform normally in spatial reversal learning tasks but to be impaired in their ability to form visual reversal sets (Gonzalez, Roberts, \& Bitterman, 1964). The present study employs what is essentially a spatial reversal problem in animals with more extensive neocortical lesions. Fish, acorticate vertebrates, have been said not to show reversal learning sets on either visual or spatial problems (Bitterman, 1965), though the generality of this finding for all species of fish and for all test situations has been challenged (Mackintosh, 1974; Warren, 1974). It nevertheless raises the possibility that with more nearly complete neodecortication the mammal's ability to form spatial reversal learning sets will also be impaired.

\section{Method}

Subjects and Surgery. The subjects were three neodecorticated and six sham-operated male Dutch rabbits with a mean body weight of $1,953 \mathrm{~g}(\mathrm{SD} \pm 271.3 \mathrm{~g})$ prior to surgery. The decortications were produced in two stages, with 24-28 weeks between operations and 25-32 weeks of postoperative recovery before testing commenced. The sham-operated animals received two 5-mm-diam trephine holes over parietal cortex, with 22-26 weeks between operations and 25-27 weeks of postoperative recovery. All surgery was carried out under chloral hydrate/pentobarbital (Equi-Thesin) anaesthesia. Neodecortication was achieved by removing the pia from the surface of the hemisphere, thereby devascularizing it and causing cortical necrosis (Meyer \& Meyer, 1971; Oakley \& Russell, 1972, 1975). So far as possible, the left hemisphere was lesioned first in half the animals and the right hemisphere first in the remainder. All subjects were housed singly and had free access to food and water until the commencement of the experiment. Table 1 summarizes interoperative and postoperative intervals, lesion size, and order of lesioning for all subjects.

\section{Apparatus}

The operant conditioning box measured $60 \times 33 \mathrm{~cm}$, had $33-\mathrm{cm}$-high walls, and was housed in a sound-attenuating chamber 
Table 1

Interoperative Intervals, Postoperative Intervals, Lesion Sizes, and Order of Lesioning of All Subjects

\begin{tabular}{ccccc}
\hline & \multicolumn{2}{c}{ Interval } & \multicolumn{2}{c}{ Lesion } \\
\cline { 2 - 3 } \cline { 5 - 5 } $\begin{array}{c}\text { Inter- } \\
\text { operative }\end{array}$ & $\begin{array}{c}\text { Post- } \\
\text { operative }\end{array}$ & Percent & Order \\
\hline \multicolumn{5}{c}{ Decorticate Subjects } \\
178 & 27 & 32 & 94 & R-L \\
224 & 24 & 25 & 94 & R-L \\
232 & 28 & 28 & 97 & L-R \\
& & Normal Subjects & $\cdot$ & \\
226 & 22 & 26 & & R-L \\
227 & 22 & 27 & & L-R \\
228 & 24 & 25 & & R-L \\
233 & 26 & 26 & & L-R \\
235 & 24 & 27 & & R-L \\
241 & 23 & 25 & & L-R \\
\hline
\end{tabular}

Note-Interoperative interval = interval (in weeks) between two stages of hemidecortication in the decorticates and between two sham operations in the normal group. Postoperative interval = interval (in weeks) between the final operation and the first day of testing in the instrumental situation. Percent lesion = lesion size expressed as the percentage of total neocortex removed. Order of lesion $=$ order in which left- $(L)$ and right- $(R)$ sided hemidecortication or sham operations were performed.

provided with two extractor fans for ventilation. Illumination within the box was from two $60-\mathrm{W}, 240-\mathrm{V}$ ceiling-mounted incandescent bulbs run at $180 \mathrm{~V}$. The animals' activity could be monitored in all parts of the box by means of a one-way vision window set in one side wall. The two manipulanda were treadles of 1-mm-thick stainless steel situated, as shown in Figure 1, on either side of a centrally located opening giving access to the food tray. Each treadle was $5 \mathrm{~cm}$ wide, protruded $5.5 \mathrm{~cm}$ into the box, and was mounted $3 \mathrm{~cm}$ above the grid floor. A downward movement of $2 \mathrm{~mm}$ on the outer edge of the treadle, requiring a force equivalent to a 12-g weight, operated a microswitch and gave rise to a small click. The operation of an electromechanical relay situated centrally behind the food tray provided a more distinct auditory signal whenever the reinforced treadle was pressed.

The tray opening was a 9-cm-square hole cut into an end wall with its lower edge $6 \mathrm{~cm}$ above the grid floor (Figure 1). This aperture was fitted with a top-hung transparent door of 1.6-mmthick perspex which closed it except for a 1-cm gap at the bottom. Reinforcement consisted of alfalfa pellets $(190 \mathrm{mg})$ dispensed automatically to the food tray. The delivery of reinforcement was signaled only by the sound of the dispenser operating and the noise of the pellet landing in the tray. A set of four counters recorded the number of reinforcements, tray door openings, and responses on each of the two manipulanda during each experimental session.

Feeding schedules. Food deprivation commenced 2 days before training in the operant box began and consisted of the complete removal of food from the home cage. Thereafter, all animals received sufficient standard laboratory diet (SG 1) after each training session to maintain their body weights between $80 \%$ and $90 \%$ of its free-feeding value.

Tray feeding and tray-door shaping. For the first 10 sessions, 20 alfalfa pellets were placed in the food tray and the animal was allowed to explore the box. Each session lasted for $20 \mathrm{~min}$ or until all the pellets had been eaten, whichever was sooner. Tray feeding was initiated with the tray door held fully open. On the session following that on which the animal first consumed all 20 pellets within $20 \mathrm{~min}$, the tray door was fully closed so that the animal had to push it open to collect its reinforcement.
Magazine training. On each of a further 10 sessions 20 alfalfa pellets were dispensed automatically into the food tray at the rate of one every $30 \mathrm{sec}$. Treadle presses and tray-door entries were monitored during these sessions to give a baseline measure for the two response types.

Treadle-press acquisition. The treadle that was activated least often during magazine training was selected for each animal to be the positive treadle $(\mathrm{T}+)$ and was programmed to deliver alfalfa pellets on a fixed ratio schedule where each treadle press was reinforced (FR 1). The other treadle $(\mathrm{T}-)$ had no effect on the delivery of reinforcement. No restriction was placed on the manner in which the treadle should be operated. Each daily session continued until 20 reinforcements had been delivered. Acquisition training was terminated when at least $90 \%$ of the animals' treadle presses had been to $T+$ on 4 out of 5 consecutive days.

Reversal training. Once the acquisition criterion had been reached, the former $\mathrm{T}+$ was made $\mathrm{T}-$ and training continued until the animal had reversed the distribution of its treadle responses to reach a criterion of $90 \%$ or more of its responses to $\mathrm{T}+$ on 2 consecutive days. This procedure was repeated until 15 reversals of the treadle-response habit had been achieved. It is perhaps worth noting that this reversal criterion is quite a stringent one. Only 20 reinforcements were allowed per session, so that for $90 \%$ of the animal's responses to be to the reinforced treadle no more than 2 could be made to the nonreinforced treadle.

Histology. When this and the subsequent experiments were completed, the animals were prepared for histology by the administration of a lethal dose of pentobarbitone sodium anaesthetic followed by intracardiac perfusion with physiological
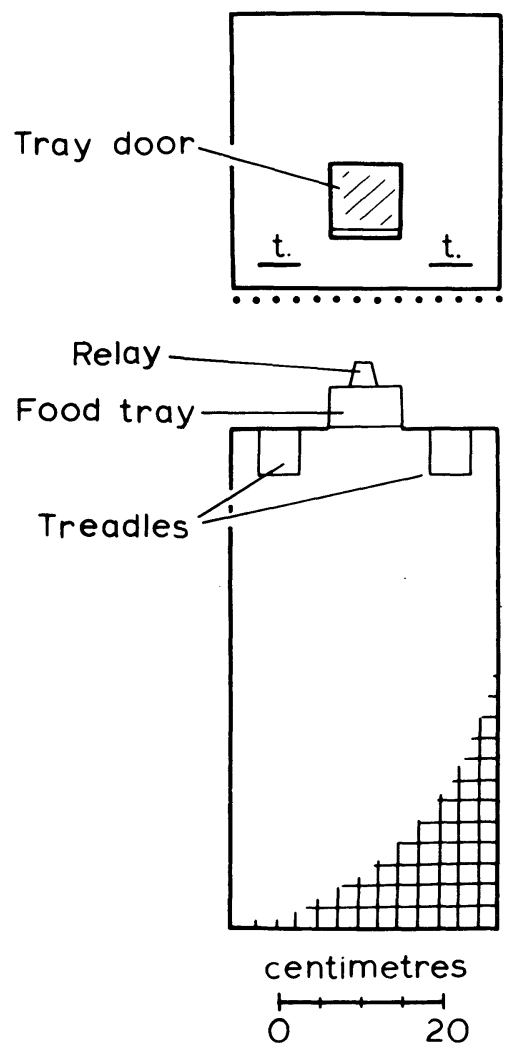

Figure 1. The operant conditioning box. Above: View of end wall from inside box showing the position of the two treadles (t) and the food tray opening. Below: Plan view. For clarity, the wire-mesh floor is indicated at bottom right only. 
saline and then a $10 \%$ solution of Formalin. Brains were removed, photographed and embedded in celloidin. Coronal sections were cut at $40 \mu$ thickness, and every 25 th section was stained with haematoxylin and eosin and mounted under glass. The lesions were drawn from the prepared sections onto a series of standard coronal sections and were then reconstructed individually onto an outline drawing of dorsal and lateral aspects of a normal rabbit brain. Estimates of lesion size were obtained by perimetry from the series of coronal sections.

Statistical analyses. Unless noted otherwise, statistical analysis was based on one-tailed Mann-Whitney U tests (Siegel, 1956).

\section{Results}

Histology. The neodecortications were similar in form and extent to lesions discussed in detail previously (Oakley \& Russell, 1976, 1977, 1978a, $1978 \mathrm{~b})$. Surface reconstructions of all three lesions are shown in Figure 2, and the percentage of neocortex removed in each case is given in Table 1 . Neocortical sparing was predominantly in frontal midline areas and occipitally in the medial ventral convexity, though one animal (178) also had a right temporal sparing. Transitional cortex was invaded in all three neodecorticates adjacent to rhinal and lateral sagittal fissures, and all showed moderate compression of caudate/putamen due to ventricular expansion. In two animals $(178,232)$, there was some evidence of superficial damage to basal ganglia on the left side. No other subcortical damage was found, nor was there any cortical damage due to the sham operations in the normal control group.

Postoperative recovery and effect of feeding schedules. After the second operation, all three

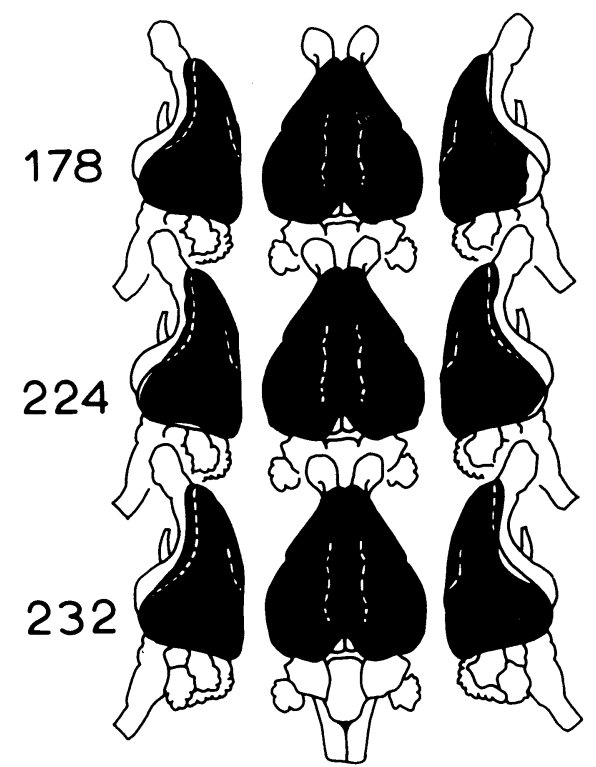

Figure 2. Reconstructions of the brains of all three neodecorticates, with the surface extent of the lesion shown in black. The rhinal fissure is shown as a solid black or dashed white line running parallel to the ventral outline in the lateral views of each brain, and the lateral sagittal fissure is shown as a white dashed line lying parallel to the midline in the dorsal view. decorticates were adipsic and aphagic. On the 4th day postoperatively, they were given liquid diet perorally by syringe and were thereafter presented with preferred foodstuffs (carrot and hay) until they returned to eating standard laboratory diet 6-9 days postoperatively. By the time training commenced, the three lesioned animals had been self-maintaining on laboratory diet and plain water for several months and had stabilized their body weights. All three maintained their fur in good condition by grooming and showed no obvious behavioral abnormalities when observed in their home environment or in an open field. When partially restrained, however, the dercorticates showed an unusually strong tendency to "race," that is, to perform vigorous but ineffective running movements, though they became as quiet as normal animals when fully restrained. This behavior had been noted in the majority of previous decorticates and was especially noticeable when restraining animals for Pavlovian conditioning.

As in previous studies (Oakley \& Russell, 1975, 1976), and in common with Braun (1975) in rats, the decorticates stabilized their body weights below those of the normal control group, though the difference in this case was not significant (mean body weights at the end of the postoperative recovery period: Normals, 2,317 $\pm 229.1 \mathrm{~g}$; Decorticates, $2,095 \pm 65.7 \mathrm{~g}$ ). During the tray-feeding and magazinetraining parts of the experiment, all animals received a standard daily ration of $10 \mathrm{~g}$ of laboratory diet, increasing to $25 \mathrm{~g} /$ day by the end of magazine training. The decorticates, despite their slightly lower postrecovery weights, responded significantly more rapidly than normals to reduced food intake and by the end of magazine training had reached $83.7 \%$ $(\mathrm{SD} \pm 1.76 \%)$ of their free-feeding weights, whereas the normals were at $86.4 \%(\mathrm{SD} \pm 1.57 \%)(\mathrm{U}=2$, $\left.\mathrm{N}^{1}=3, \mathrm{~N}^{2}=6, \mathrm{p}<.05\right)$. There was no tendency in the normal group for the lighter animals to reduce their body weights more rapidly than heavier animals, nor was there evidence of greater spillage of diet by the decorticates under deprivation conditions. These data seem to suggest a metabolic change introduced by the removal of neocortex.

Tray feeding and magazine training. In the decorticate group, animal 224 cleared all 20 pellets from the food tray on Tray Feeding Session 1, 232 did so on Session 2, and 178 not until Session 10. The normal group similarly took between 1 and 10 sessions to eat from the food tray. Introducing the tray door caused no problems for animals in either group. During the 10 days of magazine training, none of the decorticates used the dispenser click as a signal to approach the tray and retrieve the pellet, whereas three of the six normals animals were seen to utilize this discriminative stimulus and to approach the tray when reinforcement had been 
Table 2

Treadle Response Rates (Means and Standard Deviations) in Six Normal and Three Neodecorticate Rabbits on the First and Final Sessions of Magazine Training and FR1 Acquisition Training

\begin{tabular}{|c|c|c|c|c|c|c|c|c|c|c|c|c|c|c|c|c|}
\hline & \multicolumn{8}{|c|}{ Magazine Training } & \multicolumn{8}{|c|}{ FR1 Acquisition } \\
\hline & \multicolumn{4}{|c|}{ First } & \multicolumn{4}{|c|}{ Final } & \multicolumn{4}{|c|}{ First } & \multicolumn{4}{|c|}{ Final } \\
\hline & \multicolumn{2}{|c|}{ LT } & \multicolumn{2}{|c|}{ RT } & \multicolumn{2}{|c|}{ LT } & \multicolumn{2}{|c|}{ RT } & \multicolumn{2}{|c|}{$\mathrm{T}+$} & \multicolumn{2}{|c|}{$\mathrm{T}-$} & \multicolumn{2}{|c|}{$\mathrm{T}+*$} & \multicolumn{2}{|c|}{$\mathrm{T}-$} \\
\hline & $\mathbf{M}$ & SD & $\mathbf{M}$ & SD & $\mathbf{M}$ & SD & $\mathbf{M}$ & SD & $\mathbf{M}$ & SD & M & SD & $\mathbf{M}$ & SD & $\mathbf{M}$ & SD \\
\hline $\begin{array}{l}\text { Normal } \\
\text { Decorticate }\end{array}$ & $\begin{array}{l}.33 \\
.53\end{array}$ & $\begin{array}{l}.43 \\
.68\end{array}$ & $\begin{array}{l}.17 \\
.81\end{array}$ & $\begin{array}{l}.21 \\
.71\end{array}$ & $\begin{array}{l}.40 \\
.37\end{array}$ & $\begin{array}{l}.53 \\
.31\end{array}$ & $\begin{array}{l}.37 \\
.43\end{array}$ & $\begin{array}{l}.73 \\
.33\end{array}$ & $\begin{array}{r}4.45 \\
.38\end{array}$ & $\begin{array}{r}6.90 \\
.44\end{array}$ & $\begin{array}{r}.61 \\
1.11\end{array}$ & $\begin{array}{l}.78 \\
.59\end{array}$ & $\begin{array}{r}16.9 \\
8.9\end{array}$ & $\begin{array}{l}4.2 \\
3.7\end{array}$ & $\begin{array}{l}.00 \\
.11\end{array}$ & .14 \\
\hline
\end{tabular}

Note-Data are expressed in responses per minute. $L T=$ left treadle; $R T=$ right treadle; $T+=$ reinforced treadle; $T-=$ nonreinforced treadle. $\quad *$ The normal/decorticate difference here is significant ( $p<.05$, Mann-Whitney $U$, one-tailed).

signaled. Despite this clear behavioral difference between at least some of the normals and the decorticates, there were no significant differences in the rate at which the tray door was operated between normal and decorticate groups either at the beginning or end of magazine training. There were also no significant differences between the two groups in the rate at which the two treadles were activated during magazine training (see below).

Treadle-press acquisition. Treadle-press rates in responses per minute are shown in Table 2 for both groups of animals during magazine training and acquisition of FR 1 treadle pressing. During magazine training, there was no systematic difference in the rate of treadle operation across sessions or between treadles and no significant differences were seen between normals and decorticates. On the first day of FR 1 acquisition training, the normal group showed $\mathrm{T}+$ response levels that were above baseline, due to the rapid acquisition of the response by two animals. The slight elevation of $\mathrm{T}-$ scores above baseline on this day in both groups is in part due to the practice of training the animal counter to the individual treadle bias seen under magazine training. Neither of the normal/decorticate differences shown in Table 2 on the first day of FR 1 acquisition was significant, however. The difference in both groups between $\mathrm{T}+$ and $\mathrm{T}-$ response rates on the final day (Criterion Day 4) of FR 1 acquisition reflects the fact that a treadle differentiation was well established and in both groups $\mathrm{T}+$ response rates were well above baseline levels. Response rates to $\mathrm{T}+$ in the normal group were, in fact, significantly higher than those of decorticates at the end of FR 1 acquisition $\left(\mathrm{U}=2, \mathrm{~N}^{1}=3, \mathrm{~N}^{2}=6, \mathrm{p}=.048\right)$. Lower-than-normal manipulandum response rates in the decorticates is consistent with previous observations using FR schedules (Oakley, 1971; Oakley \& Russell, 1978a, 1978b), as is the fact that the normals operated the treadle by biting it while the decorticates used a variety of other means. The decorticates in this study were seen to press the treadle using nose, forefeet, and chin. One animal in the reversal part of this experiment consistently pressed the right treadle with its paws by sliding its forefeet down the end wall above the manipulandum. It operated the left treadle by depressing it with its chin. Though the decorticates did not operate the treadle with their teeth like normal animals, they were as efficient as normals in gripping their food hoppers or cage fronts in their teeth and rattling them vigorously whenever an experimenter entered the colony room around feeding time, suggesting that the difference in mode of treadle operation is not due to a motor deficit in the lesioned animals. The decorticates also took significantly more sessions $(11.0 \pm 2.9$ inclusive of criterion sessions) to acquire the differential response to $\mathrm{T}+$ than the normals $(7.0 \pm 3.4)\left(\mathrm{U}=2, \mathrm{~N}^{1}=3\right.$, $\left.\mathrm{N}^{2}=6, \mathrm{p}=.048\right)$. This difference in acquisition rates is illustrated using individual data in Figure 3.

Animals in the present situation where food delivery is reliably signaled by the noise of the dispenser operating need make no more than one tray entry per reinforcement. The number of tray entries per reinforcement is thus a convenient measure of performance efficiency (Oakley \& Russell, 1978a, 1978b). A tray/reinforcement ratio of 1 represents efficient performance. Ratios above 1 indicate unnecessary investigation of an empty food tray and ratios below 1 indicate, assuming that all reinforcements are collected, that the animal is increasing its efficiency above the minimum by allowing food pellets to accumulate before retrieving them. Tray/reinforcement ratios for both groups during magazine training and FR 1 acquisition are shown in Table 3. During magazine training, the normals were rather less efficient, though not significantly so, than the decorticates on this measure despite the fact that three normals appeared to be using the dispenser click as a signal to visit the food tray. On the first day of FR 1 acquisition training, both groups were less efficient than they had been during magazine training, with the decorticates 
Table 3

Tray/Reinforcement Ratios (Means and Standard Deviations) in Six Normal and Three Neodecorticate Rabbits on the

First and Final Sessions of Magazine Training and FR1 Acquisition Training

\begin{tabular}{|c|c|c|c|c|c|c|c|c|}
\hline & \multicolumn{4}{|c|}{ Magazine Training } & \multicolumn{4}{|c|}{ FR1 Acquisition* } \\
\hline & \multicolumn{2}{|c|}{ First } & \multicolumn{2}{|c|}{ Final } & \multicolumn{2}{|c|}{ First } & \multicolumn{2}{|c|}{ Final } \\
\hline & $\mathbf{M}$ & SD & $\mathbf{M}$ & SD & $\mathbf{M}$ & SD & $\mathbf{M}$ & SD \\
\hline $\begin{array}{l}\text { Normal } \\
\text { Decorticate }\end{array}$ & $\begin{array}{l}7.17 \\
3.91\end{array}$ & $\begin{array}{r}4.66 \\
.19\end{array}$ & $\begin{array}{l}6.68 \\
4.91\end{array}$ & $\begin{array}{l}6.41 \\
1.68\end{array}$ & $\begin{array}{r}17.4 \\
101.9\end{array}$ & $\begin{array}{l}18.2 \\
78.1 \\
\end{array}$ & $\begin{array}{r}.61 \\
2.11 \\
\end{array}$ & $\begin{array}{r}.16 \\
.92 \\
\end{array}$ \\
\hline
\end{tabular}

*The normal/decorticate difference is significant $(p<.05$, Mann-Whitney U, one-tailed).

Table 4

Number of Sessions to Reach Criterion on Original Treadle Differentiation (OTD) and Seven Subsequent Reversals of that Differentiation in Decorticate Number 224

\begin{tabular}{cccccccc}
\hline & \multicolumn{7}{c}{ Reversals } \\
\cline { 2 - 8 } OTD & 1 & 2 & 3 & 4 & 5 & 6 & 7 \\
\hline L+ & R+ & L+ & R+ & L+ & R+ & L+ & R+ \\
10 & 8 & 7 & 22 & 8 & $25^{*}$ & 8 & $20^{*}$ \\
\hline
\end{tabular}

Note $-L_{+}=$left treadle reinforced; $R_{+}^{+}=$right treadle reinforced. ${ }^{*}$ Did not reach criterion.

significantly less efficient than the normals $(U=2$, $\left.\mathrm{N}^{1}=3, \mathrm{~N}^{2}=6, \mathrm{p}=.048\right)$. By the final day of FR 1 acquisition, both groups had improved their efficiency to above baseline levels though the decorticates remained significantly less efficient than normals $\left(\mathrm{U}=1, \mathrm{~N}^{1}=3, \mathrm{~N}^{2}=6, \mathrm{p}=.024\right)$.

Treadle-press reversal. All six normals and two of the three neodecorticates were able to reverse the differential treadle response all 15 times. The third decorticate (224) reached only 7 reversals. The data for animal 224, expressed as the number of sessions taken to reach criterion, are summarized in Table 4 . The original differentiation and first two reversals progressed as in the other two decorticates, but the third reversal (right treadle reinforced, $R+v e$ ) took an unusually large number of sessions before criterion was reached. On the fifth reversal $(\mathrm{R}+\mathrm{ve}), 224$ produced $90 \%$ of its responses to $\mathrm{T}+$ on Sessions 6,11 , and 25 but not on two consecutive sessions required by the differentiation criterion. On Reversal 7 (again $\mathrm{R}+\mathrm{ve}$ ), 224 achieved no better than $65 \%$ of responses to $\mathrm{T}+$ over a 20 -session block, whereupon reversal training was abandoned for this animal. These data are taken to indicate the development of a strong and increasing left treadle preference after the first reversal and, therefore, to represent a failure to differentiate the two treadles.

Figure 3 shows sessional percentage differentiation scores during acquisition, the first three reversals, and the final three reversals for both of the decorticates that reached Reversal 15 (178 and 232), for the normal animal which showed the most improvement in reversal performance (241), and for the normal showing least improvement (228). The differentiation scores were calculated as (responses to $\mathrm{T}+$ /total responses) $\times 100$, so that a differentiation score of $0=$ all responses to the unreinforced treadle $(\mathrm{T}-), 100 \%=$ all responses to the reinforced treadle $(\mathrm{T}+), 50 \%=$ equal numbers of responses to both treadles. The two decorticates which completed reversal training took significantly more sessions over their 15 reversals (178-82 sessions; 232-110 sessions) than normals (mean 59.2 \pm 4.9 sessions) $\left(\mathrm{U}=0, \mathrm{~N}^{1}=2, \mathrm{~N}^{2}=6, \mathrm{p}=.036\right)$. As Figure 3 indicates, there were clear signs that the normal animals developed a reversal learning set, with later reversals completed more rapidly than earlier ones. On the first three reversals, the normals took a mean of $4.7(\mathrm{SD} \pm .9)$ sessions, inclusive of criterion sessions, per reversal but only $3.4(\mathrm{SD} \pm .8)$ sessions per reversal on the final three reversals. This improvement in reversal performance in the normal animals is significant (sign-test, one tie,

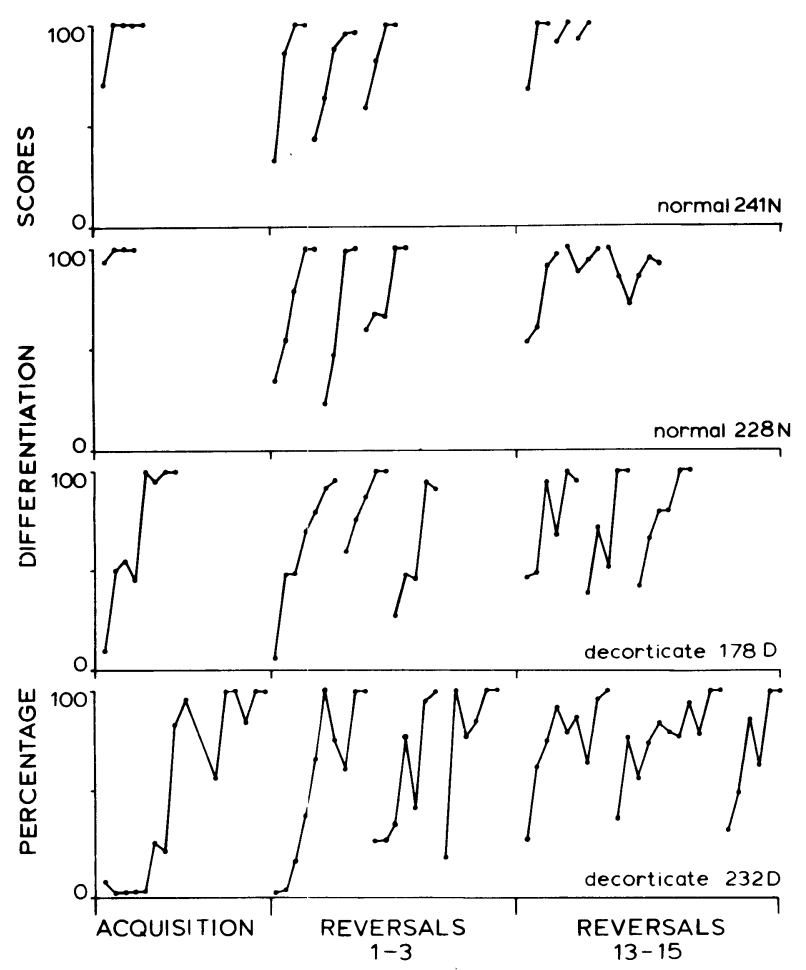

Figure 3. Sessional differentiation scores during acquisition of a two-treadle differentiation and during the first and final three reversals of that differentiation in a 15-reversal series. The top set of graphs show the performance of the normal animal which gave the best evidence of forming a reversal learning set (241), and the second set of graphs show the performance of the normal animal giving least evidence of learning set formation (228). The bottom two sets of graphs show the performance of both of the neodecorticates which completed all 15 reversals (178 and 232). The letters $N$ and $D$ after the animal numbers in this and subsequent figures denote normal and neodecorticate animals, respectively. 
$\mathrm{N}=5, \mathrm{x}=0, \mathrm{p}=.031$, one-tailed test). The two decorticates for whom complete reversal data were obtained, on the other hand, were actually taking as long or longer on average to complete each of the final three reversals (178, mean 5.7 sessions; 232, mean 8.7 sessions) than they had on the first three reversals (178, mean 5.7 sessions; 232, mean 7.3 sessions). Reversal performance can be said to have improved, irrespective of trials taken to criterion, however, if the animals learn to switch their responses progressively more readily from the former $\mathrm{T}+$ to the new $\mathrm{T}+$ on the first day of each reversal. This would be reflected in higher differentiation scores on the first sessions of later reversals. When differentiation scores on the first session of each of the 15 reversals are considered, a significant improvement is apparent in the normal group with mean differentiation scores of $55.2 \%$ ( $S D \pm 11.4)$ on the first sessions of the first three reversals, increasing to $86.4 \%$ ( $S D \pm 4.6$ ) on the first sessions of the final three reversals (sign-test, $\mathrm{N}=6, \mathrm{x}=0, \mathrm{p}=.016$, one-tailed). Interestingly, both of the decorticates which completed training also improved their firstday-of-reversal differentiation scores from low initial values on the first three reversals $(178,31.0 \%$; $232,15.3 \%$ ) to better, but still much below normal, levels on the final three reversals $(178,42.0 \%$; $232,32.0 \%$ ). This is not simply a product of the very low differentiation scores produced by both decorticates on the first session of the first reversal, as an overall improvement in first session differentiation scores is still found if Reversals 2, 3, and 4 are compared with the final three reversals.

\section{Discussion}

The neodecorticates stabilized their body weights slightly below those of normal animals and lost weight more rapidly on reduced diets, suggesting a possible metabolic change due to neocortical removal. This was not accompanied by any significant differences between normals and neodecorticates in their behavior during tray feeding, tray-door shaping, or magazine training phases of the experiment. Three of the six normal animals did, however, appear to use the dispenser click as a go signal for food-tray approach during magazine training, a behavior which was not seen in any of the decorticates. Once FR 1 was introduced, a now familiar pattern emerged. Both the normal and the neodecorticate groups increased the rate at which they pressed the reinforced treadle over sessions, though the mode of treadle operation differed between the two groups and normals responded at a higher rate than the decorticates. The normals were also more efficient than decorticates on a tray/reinforcement measure, though both groups again improved over training. More importantly, however, this first experiment showed that, though it took them more sessions than normals, neodecorticated rabbits could learn to respond differentially to one of two equally accessible treadles. Furthermore, two of the three decorticates were able to reverse that differentiation to high criterional levels at least 15 times. Unlike the normals, however, the neodecorticates showed no sign of developing a reversal learning set using a sessions-to-criterion measure, though there was evidence of a slight improvement in their differentiation performance on the first day of each reversal. It would appear, on this basis, that the formation of spatial reversal learning sets under the present conditions is seriously impaired if not abolished after removal of neocortex in rabbits. The third decorticate developed a treadle preference early in training and did not complete the reversal series. There were no obvious lesion differences that could account for this animal's performance, and it is presumed that the position habit in this decorticate represents a failure to acquire a differential treadle response and hence a failure to successfully identify the manipulanda as distinct but interchangeable entities.

\section{EXPERIMENT 2}

The two-treadle differentiation and reversal procedure was intended as a means of establishing manipulandum identification in the decorticates. This, it was predicted, would reduce their tendency to treat manipulandum and food-tray behaviors indiscriminately as parts of a global operant response and so improve the efficiency of their performance on high FRs. Previously, neodecorticates have not progressed beyond FR 8 and have maintained high levels of food-tray behavior intermixed with the manipulandum response. This second experiment investigates the performance of the three neodecorticates from Experiment 1 on increasing FR schedules.

\section{Method}

Subjects. The three decorticates and three of the normal rabbits from Experiment 1 served as subjects and were paired so that each normal member of the pair was trained on the same treadle and encountered exactly the same sequence of FR changes as its neodecorticate partner. The normal: neodecorticate pairs were 233:178, 242:232 and 241:224.

Apparatus. The apparatus was exactly as in Experiment 1, except that a cumulative recorder running at a paper speed of $10 \mathrm{~mm} / \mathrm{min}$ was used to monitor responses to the reinforced treadle and a kymograph with a paper speed of $275 \mathrm{~mm} / \mathrm{min}$ was used on some sessions to record responses to both treadles, tray-door movements, and the delivery of reinforcement.

Fixed-ratio training. All animals commenced FR training on FR 1 immediately after Reversal 15 of Experiment 1 . Reinforcement ratios were then increased on subsequent sessions at first in small steps and later in larger ones until FR 60 was reached, unless the animal's performance deteriorated, when the ratio 
was reduced progressively until the behavior recovered. Each step in the sequence covered a variable number of sessions as performance was allowed to stabilize before the next increase in FR value (see legends to Figures 4,5 , and 8 ). No restriction was placed on the manner in which the treadle should be operated. In the case of decorticates 178 and 224 , the left treadle was used for the high FR training and the right treadle for decorticate 232. Once FR 60 had been reached, the FR value was reduced to FR 10 over three sessions and stabilized

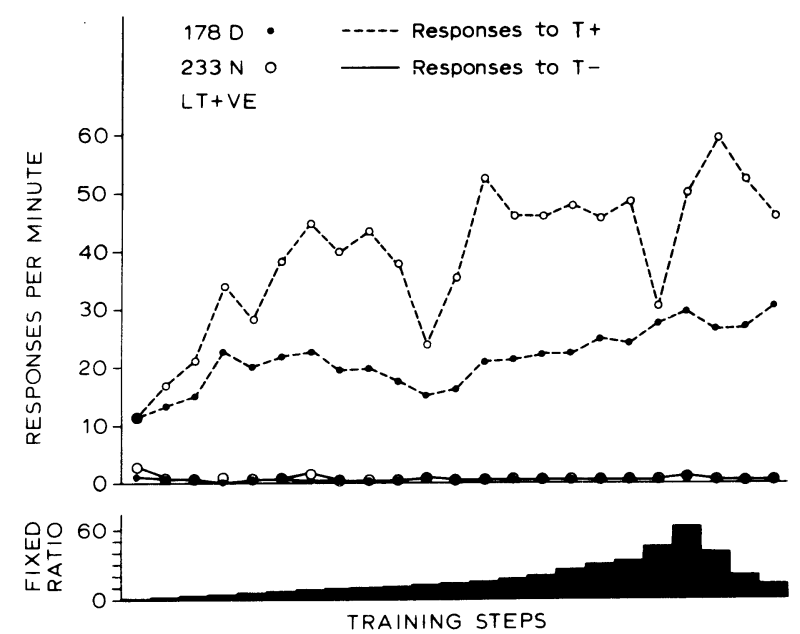

Figure 4. Above: Mean treadle response rates in responses per minute at all stages of FR training in Experiment 2 for normal 233 and neodecorticate 178. Below: Changes in FR value over training. Six sessions were given at FR 1 and at FR 60. Between these two points there was a mean of 2.4 (range 1-4) sessions per step. $L T+$ ve $=$ the left treadle was reinforced $(T+)$.
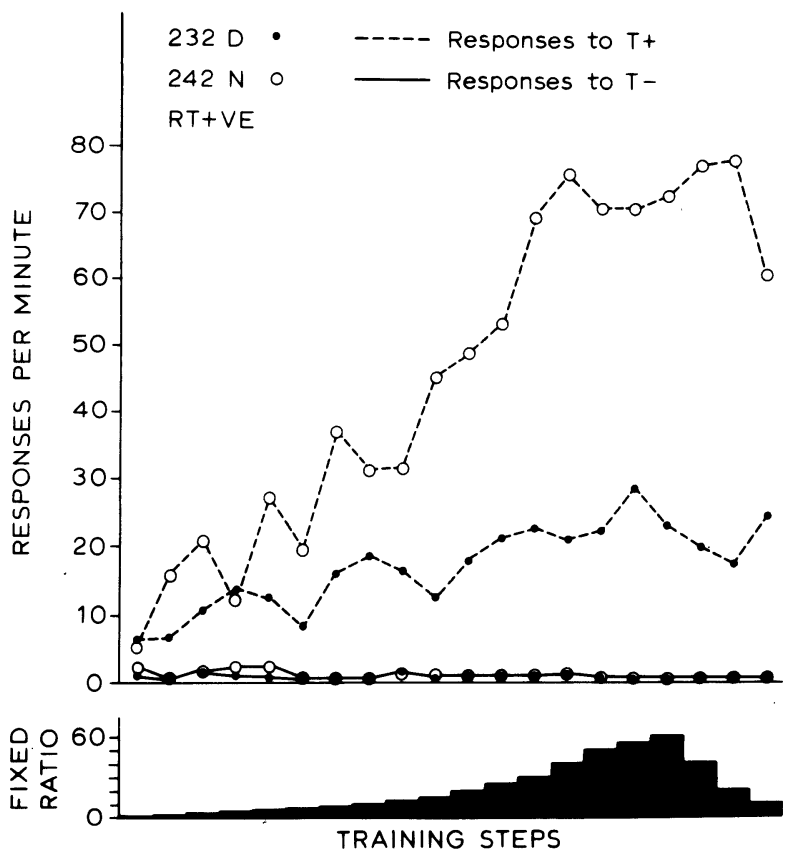

Figure 5. Above: Mean treadle response rates in responses per minute at all stages in FR training in Experiment 2 for normal 242 and neodecorticate 232. Below: Changes in FR value over training. Five sessions were given at FR 1 and 10 sessions at FR 60. Between these points, there was a mean of 2.1 (range 1-4) sessions per step. $R T+v e=$ the right treadle was reinforced $(T+)$.
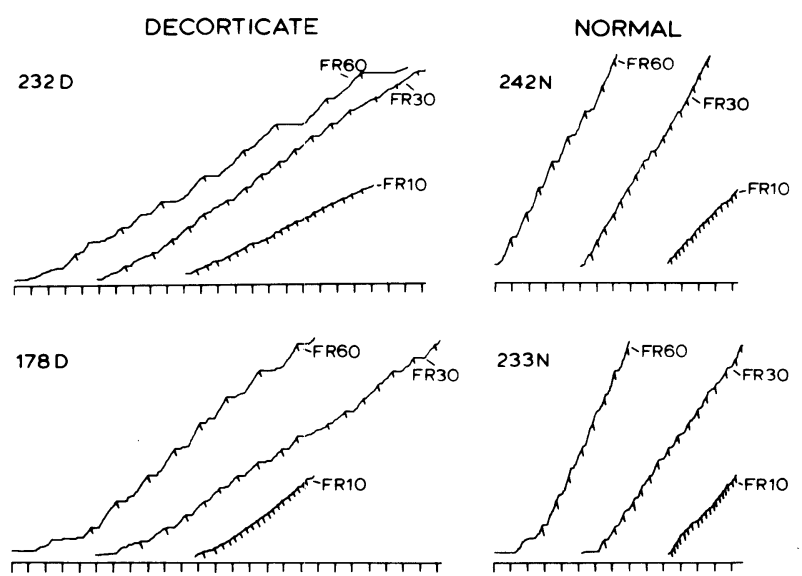

Figure 6. Sample cumulative records at FR 10, FR 30, and FR 60 for the two neodecorticate animals (232 and 178), which completed 15 reversals in Experiment 1, and their paired normal controls (242 and 233). The baseline is marked in 1-min intervals.

at that level for four to five further sessions. All sessions lasted for 20 reinforcements.

\section{Results}

Performance of decorticates which had completed 15 reversals. For clarity, the two decorticates which completed all 15 reversals in Experiment 1 will be considered first with their normal partners. Figure 4 summarizes the sequence of FR steps and response rates to $\mathrm{T}+$ and $\mathrm{T}-$ - for decorticate 178 and normal 233. The same information is given for decorticate 232 and normal 242 in Figure 5. All four animals increased their mean response rates to $T+$ over training sessions and reached FR 60 . In both pairs, the overall rates were higher in the normals than in the decorticates. Responses to $\mathrm{T}-$ were extremely rare in all four animals.

Mean sessional response rates shown in Figures 4 and 5, while indicative of general response trends, obscure the more detailed changes taking place in response topography. Increasing FRs are classically associated with increasing postreinforcement pauses and, with extended training, steady local or "running" response rates irrespective of the FR (e.g., Felton \& Lyon, 1966). Figure 6 shows sample cumulative records for all four animals at FR 10, FR30, and FR 60, from which it is apparent that both normals and neodecorticates show the expected increase in postreinforcement pause duration with increasing FR value. Taking the duration of the first visible break in responding following reinforcement, irrespective of whether it is the longest break before the next reinforcement, as a measure of postreinforcement pause duration confirms this impression (Table 5). Mean local response rates, calculated by correcting sessional response rates for changes in postreinforcement pause duration at FR 10, FR 30, and FR 60 , respectively, were $40.4,74.3$, and 87.6 responses/min for the normals and $24.0,31.5$, and 
Table 5

Showing Duration of Postreinforcement Pause in Seconds (Means and Standard Deviations) in Two Normal and Two Neodecorticate Rabbits at FR10, FR30, and FR60

\begin{tabular}{|c|c|c|c|c|c|c|c|c|c|c|c|c|c|}
\hline \multicolumn{7}{|c|}{ Normals } & \multicolumn{7}{|c|}{ Decorticates } \\
\hline \multirow[b]{2}{*}{ Subject } & \multicolumn{2}{|c|}{ FR10 } & \multicolumn{2}{|c|}{ FR30 } & \multicolumn{2}{|c|}{ FR60 } & \multirow[b]{2}{*}{ Subject } & \multicolumn{2}{|c|}{ FR10 } & \multicolumn{2}{|c|}{ FR30 } & \multicolumn{2}{|c|}{ FR60 } \\
\hline & $\mathbf{M}$ & SD & $\mathbf{M}$ & SD & $\mathbf{M}$ & SD & & $\mathbf{M}$ & SD & $\mathbf{M}$ & SD & $\mathbf{M}$ & SD \\
\hline $\begin{array}{l}233 \\
242\end{array}$ & $\begin{array}{l}2.2 \\
3.6\end{array}$ & $\begin{array}{l}1.4 \\
2.9\end{array}$ & $\begin{array}{l}8.1 \\
5.1\end{array}$ & $\begin{array}{l}7.0 \\
2.9\end{array}$ & $\begin{array}{l}14.7 \\
13.1\end{array}$ & $\begin{array}{r}17.4 \\
8.4\end{array}$ & $\begin{array}{l}178 \\
232\end{array}$ & $\begin{array}{r}6.6 \\
10.5\end{array}$ & $\begin{array}{l}5.2 \\
4.9\end{array}$ & $\begin{array}{l}21.6 \\
15.9\end{array}$ & $\begin{array}{r}10.4 \\
6.1\end{array}$ & $\begin{array}{l}39.1 \\
32.9\end{array}$ & $\begin{array}{l}15.9 \\
26.0\end{array}$ \\
\hline
\end{tabular}

Note-Data are based on measurements from cumulative records for a whole session at the stated FR value.

Table 6

Tray/Reinforcement Ratios (Means and Standard Deviations) in Two Normal and Two Neodecorticate Rabbits at FR10, FR30, and FR60

\begin{tabular}{|c|c|c|c|c|c|c|c|c|c|c|c|c|c|}
\hline \multirow[b]{3}{*}{ Subject } & \multicolumn{6}{|c|}{ Normals } & \multicolumn{7}{|c|}{ Decorticates } \\
\hline & \multicolumn{2}{|c|}{ FR10 } & \multicolumn{2}{|c|}{ FR30 } & \multicolumn{2}{|c|}{ FR60 } & \multirow[b]{2}{*}{ Subject } & \multicolumn{2}{|c|}{ FR10 } & \multicolumn{2}{|c|}{ FR30 } & \multicolumn{2}{|c|}{ FR60 } \\
\hline & $\mathbf{M}$ & SD & $\mathbf{M}$ & SD & $\mathbf{M}$ & SD & & $\mathbf{M}$ & SD & $\mathbf{M}$ & SD & $\mathbf{M}$ & SD \\
\hline 233 & 1.0 & .0 & 1.6 & .1 & 1.6 & .4 & 178 & 1.2 & .1 & 1.9 & .4 & 2.5 & 1.4 \\
\hline 242 & 1.9 & .0 & 2.7 & .4 & 2.2 & .9 & 232 & 2.3 & .0 & 3.5 & 1.1 & 2.4 & .9 \\
\hline
\end{tabular}

Note-Data are expressed as the number of food tray responses per reinforcement. All four animals completed 15 reversals in Experiment 1.

36 responses/min for the decorticates. There was thus a consistent tendency in both normals and decorticates for local treadle response rates to increase with increasing FRs, though the change in rate early in training between FR 10 and FR 30 was greater than that later in training between FR 30 and FR 60, presumably as some sort of asymptote was reached. Overall, the local response rates for the normals are approximately twice those of the decorticates, while the difference between the groups in postreinforcement pause duration in almost threefold. Throughout high FR training, the normals continued to operate the treadle with their teeth and the decorticates persisted in nose and foot pressing.

Tray/reinforcement ratios for both decorticates and normals are given in Table 6, from which it can be seen that, though these reversal pretrained decorticates are on balance slightly less efficient than normals, the differences are minimal and both groups perform at high levels of efficiency even under the highest FR value tested. Table 6 shows the number of tray entries per reinforcement but gives no idea of the distribution of the food-tray-oriented behavior. Kymograph records taken at FR 60 were used to produce response frequency histograms showing the distribution of both treadle $(\mathrm{T}+)$ and tray responses in the interval between reinforcements. The distributions obtained for both normal/decorticate pairs were similar, and Figure 7 shows representative histograms for the decorticate 232 and the normal 242. Each set of histograms covers a sample period during which 2,400 treadle responses were produced, and the base represents the interval between one reinforcement ( $R$ 1) and the next ( $R$ 2) divided into 20 equal blocks. This interreinforcement interval
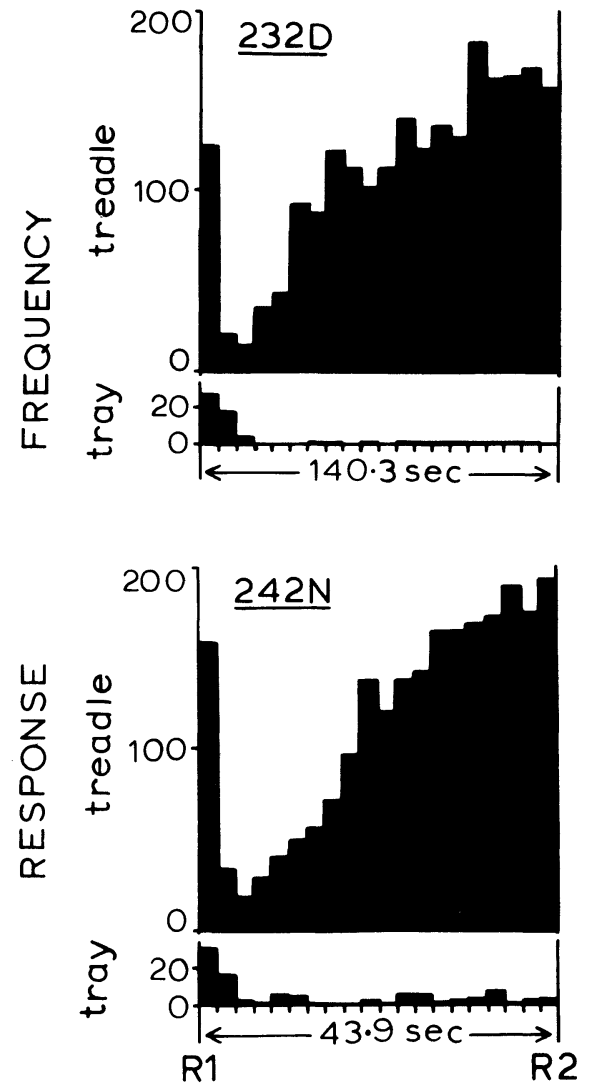

Figure 7. Distribution of tray and treadle responses within the interreinforcement interval at FR 60 in a neodecorticate (232), which completed 15 reversals in Experiment 1, and its paired normal control (242). The mean interval between consecutive reinforcements is shown in seconds beneath each pair of histograms. This figure is based on a sample of 2,400 treadle responses for each animal. $R 1=$ first reinforcement, $R 2=$ subsequent reinforcement. 


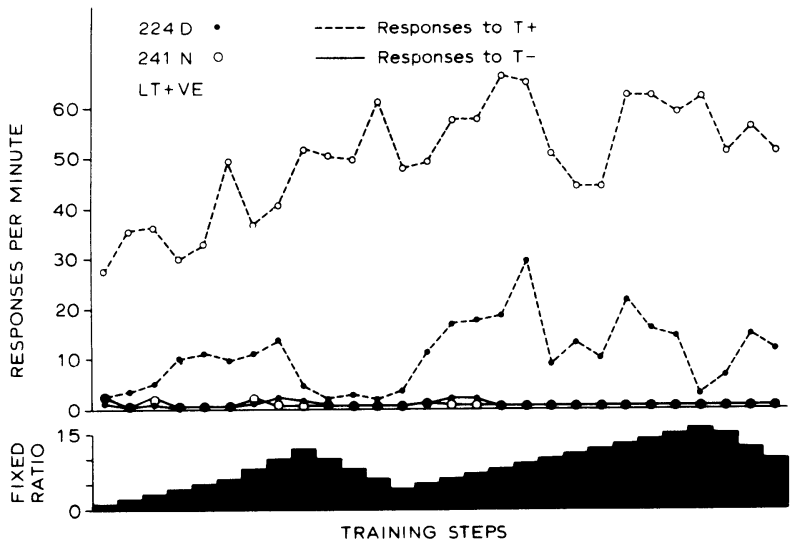

Figure 8. Above: Mean treadle response rate in responses per minute at all stages of FR training in Experiment 2 for normal 241 and neodecorticate 224. Below: Changes in FR value over training. Six sessions were given on FR 1 and six sessions on the final FR 15. Between these two points, there was a mean of 2.0 (range 1-8) sessions per step. LT + ve $=$ the left treadle was reinforced $(T+)$. The highest ratio attempted was FR 16 for one session, which is shown between two blocks of FR 15 to the right of the lower part of the figure, and was accompanied by a virtual elimination of treadle responding.

Table 7

Tray/Reinforcement Ratios (Means and Standard Deviations) in One Normal (241) and One Neodecorticate (224) Rabbit at FR5, FR10, and FR15

\begin{tabular}{lrrrrrrrr}
\hline & \multicolumn{2}{c}{ FR5 } & & \multicolumn{2}{c}{ FR10 } & & \multicolumn{2}{c}{ FR15 } \\
\cline { 2 - 3 } & M & SD & & M & SD & & M & SD \\
\hline Normal & 1.5 & .2 & & 1.9 & .4 & & 1.8 & .1 \\
Decorticate & 6.8 & 1.1 & & 16.1 & 5.2 & & 17.8 & 4.7 \\
\hline
\end{tabular}

Note-Data are expressed as the number of food tray responses per reinforcement. The decorticate (224) failed to complete 15 reversals in Experiment 1.

had a mean duration of $140.3 \mathrm{sec}(\mathrm{SD} \pm 42.9)$ in decorticate 232 and $43.9 \mathrm{sec}(\mathrm{SD} \pm 9.5)$ in normal 242 , reflecting the higher sessional response rates in the normal animal. Despite the difference in response rate, the distribution of treadle responses in both animals in Figure 7 is virtually identical. A brief "overshoot" of responses immediately following R1 is followed by a period of low response probability, corresponding to postreinforcement pauses of varying duration, and this is followed by the response run leading to reinforcement at R2. Similarly, the distribution of tray responses shows clearly that both animals were responding immediately after $\mathrm{R} 1$ and that the majority of additional tray responses (i.e., those in excess of 1 per reinforcement) were also limited to the early part of the postreinforcement pause. In fact, the decorticate member of the pair illustrated in Figure 7 was less likely than the normal to place unnecessary tray responses within the prereinforcement treadle response run.

Performance of the decorticate which failed to complete 15 reversals. The sequence of FR steps and response rates to $\mathrm{T}+$ and $\mathrm{T}-$ in decorticate 224 and its normal partner 241 are shown in Figure 8 . This decorticate initially increased its treadle response rate as the FR value increased but seemed to have an upper limit to adequate performance around FR 9-FR 10. Both attempts shown in Figure 8 to raise the FR above FR 10 resulted in the loss of treadle responding. The response was reinstated on both occasions by reducing the FR values once more. The normal member of this pair (241) performed similarly to the other normals on the same FRs, giving sessional response rates which were some four times greater than those of its paired neodecorticate on all FRs.

Table 7 shows tray/reinforcement ratios for both animals and reveals the low levels of efficiency on this score by the decorticate even on FRs 5 and 10 when it was treadle pressing reliably. The normal animal's performance is comparable to that of the previous normals shown in Table 6. Response frequency histograms constructed as before from kymograph records but based on 300 responses at FR 15, when the decorticate was treadle pressing steadily but at a low rate, are presented as Figure 9.
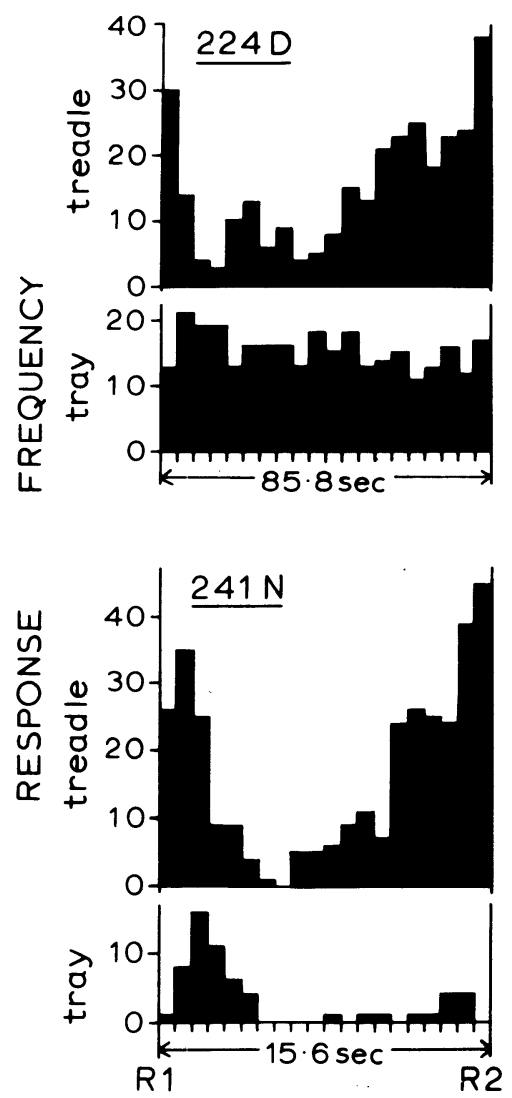

Figure 9. Distribution of tray and treadle responses within the interreinforcement interval at FR 15 in the neodecorticate (224), which failed to complete 15 reversals in Experiment 1 , and its paired normal control (241). The mean interval between consecutive reinforcements is shown in seconds beneath each pair of histograms. This figure is based on a sample of $\mathbf{3 0 0}$ treadle responses for each animal. $R 1=$ first reinforcement, $R 2=$ subsequent reinforcement. 
The treadle response distributions are similar for both animals and generally conform to the pattern described in relation to Figure 7 with an "overshoot" followed by a post reinforcement pause and then a prereinforcement response run, though the postreinforcement pause is perhaps a little cleaner in the normal animal (241). The tray response pattern of the normal is again exactly as described previously with the majority of the responses in the early postreinforcement pause period. The decorticate 224 , however, was strikingly different, with tray responses persisting at a high level throughout the interreinforcement interval. This pattern of tray responding was typical of this animal at all FRs. The mean interreinforcement interval in decorticate 224 at FR 15 was $85.8 \mathrm{sec}(\mathrm{SD} \pm 55.1$ ), and in normal 241 it was $15.6 \mathrm{sec}(\mathrm{SD} \pm 12.2)$ underlining the much greater difference in sessional response rates in these two animals compared with those of the two pairs of animals reported in the previous section.

\section{Discussion}

The decorticate which failed to complete all 15 reversals in Experiment 1 produced the sort of FR performance described for decorticated rabbits in previous studies (Oakley, 1971; Oakley \& Russell, 1978a, 1978b). In particular, response rates were approximately one-quarter those of normal animals on the same ratios, tray behavior continued throughout the entire interreinforcement interval, and ratios above FR 10 resulted in a drop in response ratesa ceiling close to the FR 8 suggested by the earlier studies. The two decorticates which did complete reversal training, however, responded to the treadle with mean response rates closer to, but still below, those of normal controls, limited their tray behavior to the postreinforcement pause exactly as normals, and showed no sign of a ceiling to the FR value at which they would work. One interpretation of this result is that the two normals successfully pretrained on treadle reversal had learned to identify, or pay attention to, the manipulandum, and so were able to separate food-tray- and treadle-related components of their performance. This, in turn, by allowing treadle activity to continue uninterrupted, not only increased overall response rates, but enabled higher FR response runs to be achieved. The neodecorticate which failed to complete the reversal pretraining because of a position habit, on the other hand, had presumably not identified the treadle in isolation from a particular spatial relationship with the food tray. This animal, like those in the previous studies, thereafter failed on FR training to respond separately to the treadle and wove its treadle response into a complex behavior which involved tray investigation as an undifferentiated component of a response chain leading to reinforcement. It is perhaps significant that the tray response rates in this and previous nonpretrained neodecorticates were, in fact, higher than treadle response rates. Persistent tray investigation not only reduces the rate of treadle responding that can be achieved but also means that repetitive treadle responses cannot be performed as a single unit, so perhaps accounting for the low FR ceiling.

Alternatively, it might be that the FR acquisition failure in decorticate 224 is simply another reflection of a general learning deficit which also prevented acquisition of the treadle reversal response and which might be due to an undetected lesion difference. It would be necessary to assume, however, that this also accounted for similar performances on FR in the previous neodecorticates and that, in fact, it was the lesions of the two successful animals which were different from all previous ones produced in the same way. No such difference in lesion extent or distribution which distinguished both decorticates 178 and 232 from their predecessors was found. At present, then, the difference in pretraining both within this experiment and between this study and the previous ones seems to be the factor most probably responsible for the differences in FR performance observed. There is a clear need, however, to check this interpretation with a selfcontained study where both the extent and nature of the pretraining is systematically varied. As the neodecorticated rabbits unsuccessfully trained on high FR schedules in the previous studies had extensive prior experience of Pavlovian nictitating membrane conditioning, it would appear that the pretraining should be specific to the instrumental response to be effective. The data of 224 suggests further that training should not only be specific but should also be successful in establishing a differentiated response to the manipulandum per se.

As to other features of neodecorticate performance on high FRs, it should be noted that all the decorticates in this study, as in previous ones, used means other than the normal biting method to operate the treadle. Similarly, all the neodecorticates so far tested irrespective of pretraining have produced treadle response distributions within the interreinforcement interval which were similar to those of normal animals. The sequence described of "overshoot," postreinforcement pause, and prereinforcement response run thus seems, like Pavlovian conditioning, to be independent of neocortical mechanisms (Oakley \& Russell, 1977). This would be expected if, for instance, the interreinforcement treadle response topography reflected Pavlovian influences on response probability over time.

\section{EXPERIMENT 3}

During magazine training, it was noted that half the normal animals used the dispenser click as a go signal for food-tray approach whereas none of the decorticates appeared to do so. This final experiment 
investigates go/no-go learning in the three neodecorticates from Experiment 2. Using partially neodecorticated rats $(47 \%-78 \%$ of neocortex removed), Bloch and Bello (1974) have reported that a go/no-go contingency using a leverpress response was learned if the differential signal was introduced at the outset of training but not if the go/no-go schedule followed the establishment of go responding. As all of the present animals learned the treadle press under go conditions before the introduction of the go/no-go, they would not be expected, on the basis of Bloch and Bello's data, to develop the appropriate behavior.

\section{Method}

Subjects. The subjects were the three neodecorticate and the three normal animals from Experiment 2 plus an additional normal animal (228) which had been a subject in Experiment 1 and had received the same FR training program as decorticate 232 and normal 242 in Experiment 2.

Apparatus. The apparatus was as in Experiment 2.

Go/no-go training. All subjects had been brought onto FR 10 and stabilized at that ratio for four to five sessions at the end of Experiment 2. The go/no-go training was on the $\mathrm{T}+$ established in Experiment 2, and consisted of a multiple (FR 10, extinction) schedule. The other treadle $(\mathrm{T}-$ ) remained in the box, and responses to it were recorded but never reinforced-a simple extinction schedule. Each 20 -min session commenced with 2 min of extinction with the houselights on, followed by 2 min of FR 10 with the houselights turned off. This sequence was repeated until five light and five dark periods had elapsed. All previous FR training in these animals had been conducted with houselights on so they had now to learn to inhibit an established tendency to respond during the light as well as to learn to respond during the dark period. The decorticate 232 had received 12 sessions of go/no-go training before it was perfused to secure its brain for histology; the rest had received 20 sessions before perfusion.

\section{Results}

Perhaps somewhat surprisingly, in view of the reversal training that all these animals received in Experiment 1, responding to $\mathrm{T}-$ was negligible in all animals at all stages of training, even during those periods when $\mathrm{T}+$ was under extinction conditions on the go/no-go schedule. The performance of all subjects on the reinforced treadle $(T+)$ is summarized in the form of go/no-go scores in Figure 10. These scores were obtained for each animal by dividing the number of responses to $\mathrm{T}+$ during go periods by the total number of responses to $\mathrm{T}+$ over the whole session. A score of 1 therefore indicates that all responses were made during go periods, a score of 0 indicates that all responses were made during no-go periods, and a score of .5 shows that equal numbers of responses were made during both go and no-go periods. On the first session, all seven animals produced scores below .5 as they responded more vigorously during the light (no-go) phase than during darkness, probably reflecting the fact that their original training had been under light conditions. There was no significant

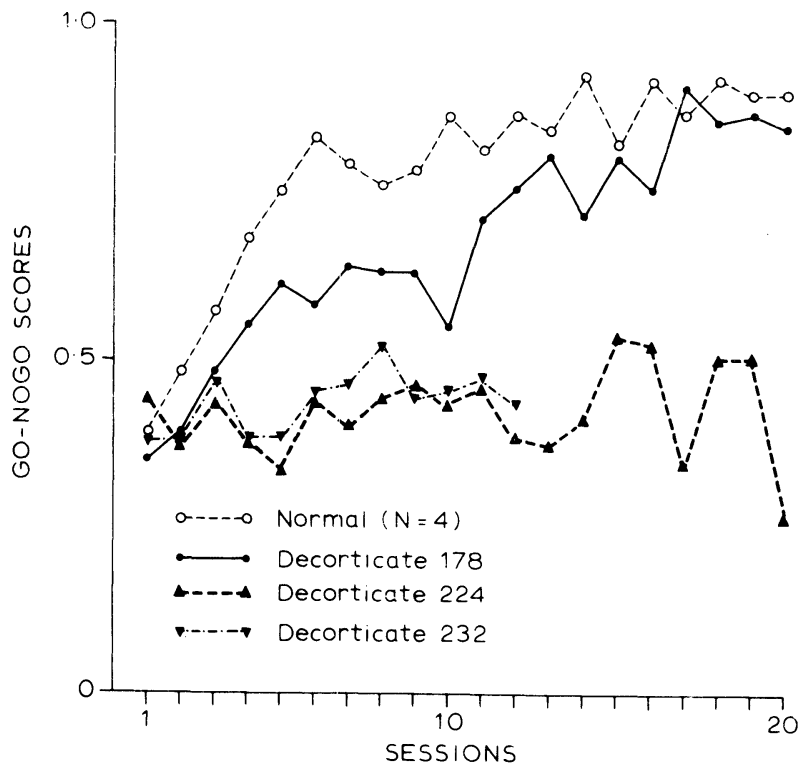

Figure 10. Go/no-go scores for four normal and three neodecorticate rabbits in Experiment 3. The normal data are shown as group curves and the decorticate data as individual curves.

difference in go/no-go scores between normals and neodecorticates in Session 1. On subsequent sessions, the normals as a group rapidly acquired the appropriate response pattern, reaching a mean score of .8 by Session 6 . Of the decorticates, two $(224$ and 232) failed to show any improvement over their original scores, whereas the third (178) eventually reached normal levels of performance. Even in 178, however, acquisition was retarded with go/no-go scores not reaching .8 until Session 13 . The go/no-go performance of the decorticates in Session 12, the last session in which all three were tested, was significantly poorer than that of normals in the same session (mean go/no-go scores in Session 12: normals, $.87 \mathrm{SD} \pm .07$; decorticates, $.52 \mathrm{SD} \pm .16 ; \mathrm{U}=0$, $\left.\mathrm{N}^{1}=3, \mathrm{~N}^{2}=4, \mathrm{p}=.028\right)$. Treadle $(\mathrm{T}+)$ response rates in go and no-go phases on the first and final sessions of training are shown in Table 8 for all

Table 8

Treadle Response Rates ( $\mathrm{T}+$ ) During the Go and No-Go Phases of the First and Final (20th) Training Sessions in Four Normal and Three Neodecorticate Rabbits

\begin{tabular}{lrrrrrr}
\hline & \multicolumn{3}{c}{ Normals } & & \multicolumn{3}{c}{ Decorticates } \\
\cline { 7 - 7 } & M & SD & & 178 & 224 & 232 \\
\hline GO Response Rates & & & & & \\
$\quad$ First Session & 38.3 & 22.0 & & 29.6 & 14.5 & 16.8 \\
$\quad$ Final Session & 52.9 & 4.9 & & 28.4 & 4.5 & $19.7^{*}$ \\
NOGO Response Rates & & & & & \\
$\quad$ First Session & 58.9 & 24.7 & & 54.8 & 18.3 & 27.1 \\
$\quad$ Final Session & 6.5 & 4.1 & & 5.3 & 12.6 & $25.9^{*}$ \\
\hline
\end{tabular}

Note-Data are presented as responses per minute. The normal data are shown as a mean and a standard deviation; the decorticate data are shown as individual mean response rates.

${ }^{*}$ Session 12. 
animals. Over training, the normals increased their go response rates slightly while markedly reducing no-go rates, producing consistently high go/no-go scores. The neodecorticates failed to show a consistent pattern, so individual data are given in Table 8, which should be read in conjunction with Figure 10. Decorticate 178 changed its go response rate very little over training but, like the normals, reduced it no-go response rate sharply to give high go/no-go scores. Decorticate 224 reduced both go and no-go rates but, with the greater of these reductions being in the go component, final go/no-go scores were low. Decorticate 232 changed neither its go nor its no-go response rates substantially over the 12 sessions for which it was tested, and so retained its initial low go/no-go score.

\section{Discussion}

Apart from the fact that the decorticate 178 was the only one with a sparing, albeit small and unilateral, of temporal neocortex, there appear to be no clear differences in lesion size or past performance history to explain why this animal should acquire the go/no-go response while the other two neodecorticates did not. What is clear from these data, however, is that neodecortication significantly retards the acquisition of go/no-go performance under the present conditions, though it does not appear to prevent it completely in all animals. Any simple interpretation of this result in terms of a general impairment of inhibitory control of responses is unacceptable in view of the generally superior to normal performance of neodecorticates in Pavlovian situations involving the inhibition of responses to a former positive conditional stimulus under reversal conditions (Oakley \& Russell, 1974, 1975). It also contrasts with the ability of neodecorticates to produce more complete suppression of responding to the nonreinforced conditional stimulus than normals in a Pavlovian light/tone differentiation (Oakley \& Russell, 1976, 1977). The present go/no-go data, along with the similar results of Bloch and Bello (1974), are consistent, however, with the longer training needed for 15 reversals by decorticates in Experiment 1 of the present study in suggesting that inhibitory control over previously established instrumental responses may be impaired by neodecortication, producing perseverative errors similar to those associated with frontal lesions in subprimates (Brutkowski, 1965; Lawicka, 1957; Maher, 1956).

\section{GENERAL DISCUSSION}

The high levels of efficient FR performance achieved by the neodecorticates successfully pretrained on reversal learning suggests that the primary deficit in these animals may not be in forming appropriate associations but may lie in such factors as identifying or paying attention to revelant aspects of the environment. These data thus seem to undermine the proposition that instrumental learning is dependent in mammals on intact neocortex (DiCara, Braun, \& Pappas, 1970; Oakley \& Russell, 1972; Russell, 1966, 1971). The treadle-press response for food used in the present experiments conforms to the usual operational definition of an instrumental learning paradigm. This is, however, insufficient evidence on which to conclude that instrumental (responsereinforcer; R-S*) learning has been shown in the absence of neocortex. It is possible that FR performance in the neodecorticates, the normal animals, or in both is mediated by implicit Pavlovian (stimulusreinforcer; S-S*) contingencies as a form of signtracking or autoshaping (Hearst, 1975; Hearst \& Jenkins, 1974). In the present situation, for instance, a close-up view of one treadle is reliably followed by the delivery of food and may thus serve as a self-presented conditional stimulus. The present experiment does not provide sufficient evidence for a final conclusion as to the relative contribution of S-S* and R-S* processes to the performance of either group of animals. Nevertheless, three points arise from these data, and all point tentatively to the conclusion that the performance of the neodecorticates cannot be explained on the basis of Pavlovian S-S* associations alone. First, Experiment 3 indicated that the neodecorticates had difficulty with response inhibition whereas previous studies using traditional Pavlovian procedures have reliably found no impairment of inhibition in the absence of neocortex. Secondly, while S-S* processes may account for the establishment of treadle responses early in training, it is less clear that they are adequate to account for efficient FR 60 performance without assuming some contribution from R-S* associations. Finally, it has been noted that the manipulandum response resulting from Pavlovian sign-tracking is similar in form to the response elicited by the reinforcer (Hearst, 1975; Hearst \& Jenkins, 1974). In the present studies, however, the neodecorticates always retrieved pellets from the food tray with their mouths but never used their mouths to operate the treadle. Some resolution between S-S* and R-S* processes is possible using an autoshaping paradigm with a negative automaintenance (omission) procedure, and preliminary work on test procedures that will be used to evaluate the performance of neodecorticated and normal animals has recently been completed (Oakley \& Davey, 1977).

While interpretive problems clearly remain, it is nonetheless evident that under the conditions of the present study neocortex is not required for efficient performance on FR schedules as high as FR 60 in a free-operant situation. This represents evidence of a greater degree of subcortical plasticity than is 
commonly assumed in neodecorticated mammals in operationally defined instrumental learning situations (Berlucchi \& Buchtel, 1975; Bjursten, Norrsell, \& Norrsell, 1976; Buchwald \& Brown, 1973; Oakley \& Russell, 1978a; Russell, 1971). Insofar as the treadle reversal training served to improve manipulandum identification, the present results are consistent with the prediction made on the basis of an earlier study (Oakley \& Russell, 1978b) that such a training program would enhance subsequent FR performance.

\section{REFERENCES}

Berlucchi, G., \& Buchtel, H. A. Some trends in the neurological study of learning. In M. S. Gazzaniga \& C. Blakemore (Eds.), Handbook of psychobiology. London: Academic Press, 1975. Pp. 481-498.

Bitterman, M. E. Phyletic differences in learning. American Psychologist, 1965, 20, 396-410.

BuUrsten, L.-M., Norrsell, K., \& Norrsell, U. Behavioural repertory of cats without cerebral cortex from infancy. Experimental Brain Research, 1976, 25, 115-130.

Bloch, S., \& Bello, M. Differential instrumental learning with food reward after extensive neocortical lesions in rats. Acta Neurobiologiae Experimentalis, 1974, 34, 603-613.

Bloch, S., \& LAGARRIGUE, I. Cardiac and simple avoidance learning in neodecorticate rats. Physiology \& Behavior, 1968, 3, 305-308.

BRAUn, J. J. Neocortex and feeding behavior in the rat. Journal of Comparative and Physiological Psychology, 1975, 89, 507-522.

BROMILEY, R. B. Conditioned responses in a dog after removal of neocortex. Journal of Comparative and Physiological Psychology, 1948, 41, 102-110.

Brutkowski, S. Functions of prefrontal cortex in animals. Physiological Review, 1965, 45, 721-746.

BuchwAld, J. S., \& BRown, K. A. Subcortical mechanisms of behavioural plasticity. In J. D. Maser (Ed.), Efferent organization and the integration of behavior, London: Academic Press, 1973. Pp. 99-136.

Chopin, S. F., \& Buerger, A. A. Graded acquisition of an instrumental avoidance response by the spinal rat. Physiology \& Behavior, 1975, 15, 155-158.

Culler, E., \& Metrler, F. A. Conditioned behaviour in a decorticate dog. Journal of Comparative Psychology, 1934, 18, 291-303.

DiCara, L. V., Braun, J. J., \& Pappas, B. A. Classical conditioning and instrumental learning of cardiac and gastrointestinal responses following removal of neocortex in the rat. Journal of Comparative and Physiological Psychology, 1970, 73, 208-216.

ENSER, L. D. A study of classical nictitating membrane conditioning in neodecorticate, hemidecorticate and thalamic rabbits. Doctoral dissertation, University of Iowa, 1976.

Felton, M., \& Lyon, D. O. The post-reinforcement pause. Journal of the Experimental Analysis of Behavior, 1966, 9, 131-134.

Flood, N. C., Overmier, J. B., \& Savage, G. E. Teleost telencephalon and learning: An interpretive review of data and hypotheses. Physiology \& Behavior, 1976, 16, 783-798.

Girden, E., Mettler, F. A., Finch, G., \& Culler, E. Conditioned responses in a decorticate dog to acoustic, thermal and tactile stimulation. Journal of Comparative Psychology, 1936, 21, 367-385.

Goltz, F. M. Der Hund ohne Grosshirn. Pflügers Archiv für die Gesamte Physiologie, 1892, 51, 570-614.

Gonzalez, R. C., Roberts, W. A., \& Bitterman, M. E. Learning in adult rats with extensive cortical lesions made in infancy. American Journal of Psychology, 1964, 77, 547-562.

Hearst, E. Pavlovian conditioning and directed movements. In G. H. Bower (Ed.), The psychology of learning and motivation:
Advances in research and theory (Vol. 9). New York: Academic Press, 1975. Pp. 215-262.

HeArst, E., \& Jenkins, H. M. Sign-tracking: The stimulusreinforcer relation and directed action. Austin, Texas: Psychonomic Society, 1974.

Huston, J. P., \& Borbély, A. A. The thalamic rat: General behavior, operant learning with rewarding hypothalamic stimulation, and effects of amphetamine. Physiology \& Behavior, 1974, $12,433-448$.

LAWICKA, W. M. The effect of prefrontal lobectomy on the vocal conditioned reflexes in dogs. Acta Biologiae Experimentalis, 1957, 17, 317-325.

Lebedinskaia, S. I., \& Rosenthal, J. S. Reactions of a dog after removal of the cerebral hemispheres. Brain, 1935, 58, 412-419.

MACKIntosh, N. J. The psychology of animal learning. London: Academic Press, 1974.

MAHER, B. A. Anticipatory and perseverative errors following frontal lesions in the rat. Journal of Comparative and Physiological Psychology, 1955, 48, 102-105.

McConnell, J. V., \& JACOBSON, A. L. Learning in invertebrates. In D. A. Dewsbury \& D. A. Rethlingshafer (Eds.), Comparative psychology: A modern survey. Tokyo: McGraw-Hill Kogakusha, 1974. Pp. 429-470.

MEYER, P. M., \& MEYER, D. R. Neurosurgical procedures with special reference to aspiration lesions. In R. D. Myers (Ed.), Methods in psychobiology (Vol. 1). London: Academic Press, 1971. Pp. 91-130.

OAKLEY, D. A. Instrumental learning in neodecorticate rabbits. Nature [New Biology], 1971, 233, 185-187.

OAKLEY, D. A., \& DAVEY, G. C. L. Differential control of autoshaping in the rat: Contrast effects and reversal learning. IRCS Medical Science, 1977, 5, 519.

OAKLEY, D. A., \& Russell, I. S. Mass action and Pavlovian conditioning. Psychonomic Science, 1968, 12, 91-92.

OAKLey, D. A., \& Russell, I. S. Neocortical lesions and Pavlovian conditioning. Physiology \& Behavior, 1972, 8, 915-926.

OAKLEY, D. A., \& Russell, I. S. Differential and reversal conditioning in partially neodecorticate rabbits. Physiology \& Behavior, 1974, 13, 221-230.

OAKLey, D. A., \& Russell, I. S. Role of cortex in Pavlovian discrimination learning. Physiology \& Behavior, 1975, 15, 315-321.

OAKLEY, D. A., \& Russell, I. S. Subcortical nature of Pavlovian differentiation in the rabbit. Physiology \& Behavior, 1976, 17, 947-954.

OAKLeY, D. A., \& Russell, I. S. Performance of neodecorticated rabbits in a free-operant situation. Physiology \& Behavior, 1978, 20, 157-170. (a)

OAKLEY, D. A., \& Russell, I. S. Manipulandum identification in operant behavior in neodecorticate rabbits. Physiology \& Behavior, 1978, 21, in press. (b)

Pavlov, I. P. Conditioned reflexes (Translated and edited by G. V. Anrep). London: Oxford University Press, 1927.

Russell, I. S. Animal learning and memory. In D. Richter (Ed.), Aspects of learning and memory, London: Heinemann, 1966. Pp. 121-171.

Russell, I. S. Neurological basis of complex learning. British Medical Bulletin, 1971, 27, 278-285.

SIEGEL, S. Nonparametric statistics for the behavioral sciences. New York: McGraw-Hill, 1956.

W ARREN, J. M. Learning in vertebrates. In D. A. Dewsbury \& \& D. A. Rethlingshafer (Eds.), Comparative psychology: $A$ modern survey. Tokyo: McGraw-Hill Kogakusha, 1974, Pp. 471-509.

Zeliony, G. P., \& Kadykov, B. I. Contribution to the study of conditioned reflexes in the dog after cortical extirpation. Med. exp. Kharkov, 1938, 3, 31-34. Psychological Abstracts, 1938, 12 , No. 5829.

(Received for publication February 14, 1978; revision accepted August 23, 1978.) 